\title{
Abnormal oscillation modes in a waning light bridge
}

\author{
Ding Yuan and Robert W. Walsh
}

\begin{abstract}
Jeremiah Horrocks Institute, University of Central Lancashire, Preston PR1 2HE, UK
e-mail: DYuan2@uclan.ac.uk
\end{abstract}

Received 6 July 2016 / Accepted 11 September 2016

\begin{abstract}
Context. A sunspot acts as a waveguide in response to the dynamics of the solar interior; the trapped waves and oscillations could reveal its thermal and magnetic structures.

Aims. We study the oscillations in a sunspot intruded by a light bridge, and the details of these oscillations could reveal the fine structure of the magnetic topology.

Methods. We used the Solar Dynamics Observatory/Atmospheric Imaging Assembly data to analyse the oscillations in the emission intensity of light bridge plasma at different temperatures, and we investigated their spatial distributions.

Results. The extreme ultraviolet emission intensity exhibits two persistent oscillations at five-minute and sub-minute ranges. The spatial distribution of the five-minute oscillation follows the spine of the bridge, whereas the sub-minute oscillations overlap with two flanks of the bridge. Moreover, the sub-minute oscillations are highly correlated in spatial domain, however, the oscillations at the eastern and western flanks are asymmetric with regard to the lag time. In the meantime, jet-like activities are only found at the eastern flank.

Conclusions. Asymmetries in the form of oscillatory pattern and jet-like activities are found between two flanks of a granular light bridge. Based on our study and recent findings, we propose a new model of twisted magnetic field for a light bridge and its dynamic interactions with the magnetic field of a sunspot.
\end{abstract}

Key words. Sun: atmosphere - Sun: corona - Sun: oscillations - sunspots

\section{Introduction}

A sunspot is a localized dark and cool area on the Sun with strong magnetic field that suppresses the dynamic motions of the hot gas. Waves and oscillations are established within a sunspot, which act as a waveguide in response to the dynamics and turbulence of the solar interior (see a comprehensive review by Khomenko \& Collados 2015). Analysis of the spatial and height distributions of the oscillations can reveal the magnetic (Reznikova \& Shibasaki 2012; Yuan et al. 2014b,a; Jess et al. 2013, 2016) and thermal structures of a sunspot (Zhugzhda 2008; Botha et al. 2011; Snow et al. 2015; Yuan et al. 2016; Chae \& Goode 2015; Kwak et al. 2016). An isolated sunspot could be assumed to have an expanding axisymmetric magnetic field; inhomogeneities, such as light bridges, umbral dots, are normally observed at a variety of scales (see a review by Borrero \& Ichimoto 2011). These inhomogeneities could modify the distribution of the oscillation frequency (Jess et al. 2012) and even even cause abnormal frequency changes (Yuan et al. 2014a; Su et al. 2016). Therefore, they are excellent objects to test the feasibility and robustness of the presumed theory and to explore the induced extra dynamics, for example reconnections, heating, flows, and convections.

This study concentrates on one such inhomogeneity: the light bridge. A light bridge is usually formed as a lower atmospheric structure in nascent or decaying sunspots. It divides an umbra into separate cores (Sobotka et al. 1994; Lagg et al. 2014), and convection, which is normally suppressed by the strong magnetic field of a sunspot, is partially restored (Rimmele 1997; Schüssler \& Vögler 2006). Consequently, upflows are usually observed at the spine of a bridge, and downflows (or return flows) are observed at the two flanks (Louis et al. 2009). The downflows drag the umbral field downwards and may even cause polarity reversal (Lagg et al. 2014). Recent numerical simulations reveal that light bridges could be formed as the result of an emerging bipole field (Toriumi et al. 2015a,b). Magnetic null points formed at a flank of a light bridge could be preferred locations for magnetic reconnection (Cheung \& Cameron 2012). Louis et al. (2014) found that one flank of a bridge supports prevailing chromospheric jet activities caused mainly by magnetic reconnection. This implies that the magnetic field of a light bridge may be twisted along its length and the field may only shape a magnetic null point at one flank.

Yuan et al. (2014a) reported the first detection of five-minute oscillation at a light bridge observed at the chromospheric height. Su et al. (2016) suggested that the inference counterstream three-minute umbral wave might be the source of the five-minute oscillations at light bridge. Yang et al. (2015) detected transverse oscillation of a wing of a light bridge at a fiveminute range and termed it a "light wall oscillation". This kind of oscillation could be detected as periodic intensity variations if the motion is along the line of sight (Yuan \& Van Doorsselaere 2016a,b). At the current stage, the source of the five-minute oscillations at light bridges still awaits to be revealed.

In this paper, we present the first detection of two abnormal oscillation modes in a waning light bridge. These modes are anomalous in comparison with the conventional sunspot oscillations (Jess et al. 2016; Khomenko \& Collados 2015; Yuan et al. 2014b), and are uniquely associated with granular light bridges. The spatial distributions of the oscillations reveal the fine structure of the bridge, a potential heating mechanism that sustains a significant portion of the plasma at coronal temperature, and 


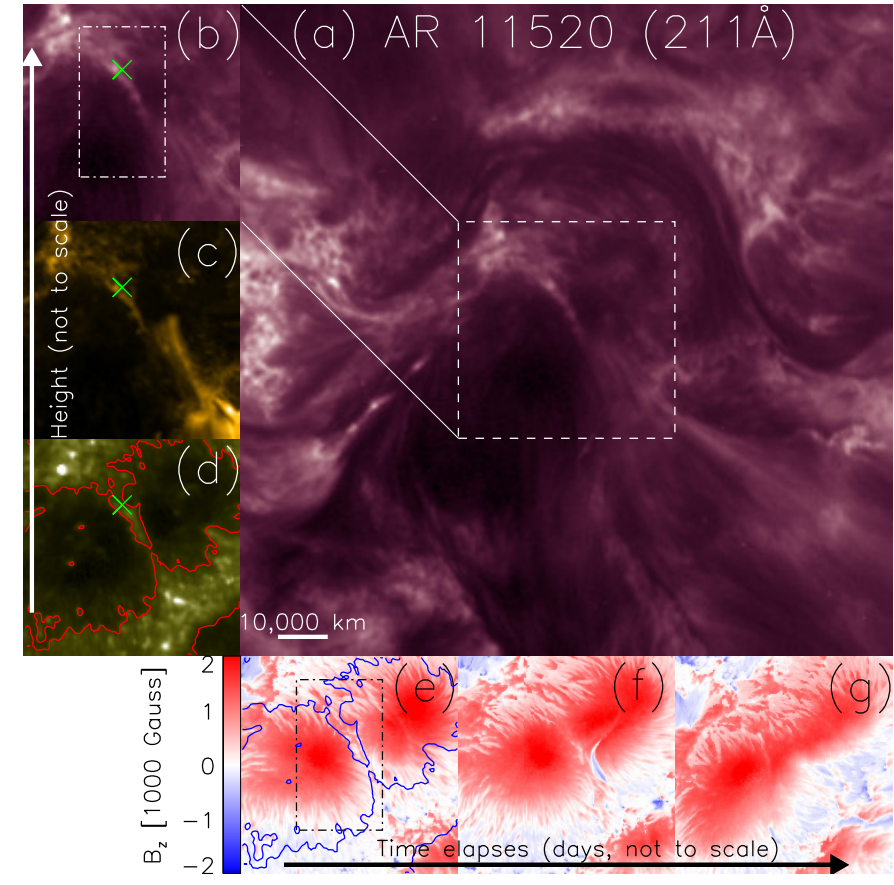

Fig. 1. a) Field of view (FOV) of active region AR 11520 observed in the AIA $211 \AA$ channel on 11 July 2012. The active region is relatively clear of coronal loops and other complex coronal structures, therefore, the light bridge is easily identifiable. Smaller FOV (as enclosed in the dashed rectangle in a)) observed in the AIA b) $211 \AA(2000000 \mathrm{~K})$, c) $171 \AA(600000 \mathrm{~K})$, and d) $1600 \AA(5000 \mathrm{~K})$ channels, respectively. The green crosses label the pixel where time series were extracted and analysed in Fig. 2. The dot-dashed rectangle in b) indicates the area where power maps are calculated and analysed in Fig. 3. e)-g) Evolution of the local normal magnetic field measured by Hinode SOT/SP on 11,13 , and 14 July 2012, respectively. The contours in d) and e) show the light bridge structure at upper photosphere $(1600 \AA)$ as a reference.

a possible magnetic ablation process that eventually leads to the disappearance of the bridge. We present the method in Sect. 2 and the results and discussions in Sect. 3.

\section{Method}

\subsection{Data reduction}

This study focusses on active region AR 11520 located at the southern hemisphere on 11 July 2012. The main spot has a positive polarity and is formed of two umbral cores separated by a light bridge (Fig. 1). The bridge under examination is a photospheric (or granular) light bridge (Muller 1979; Sobotka et al. 1994); it has a central lane with a relatively stronger magnetic field component $\left(B_{z}\right)$ along the local normal direction than either flank (Figs. 1e and 4). The central lane is usually dark if observed in the $G$-band (Rimmele 2008). Upflows were detected at the spine of a bridge and surrounded by weaker downflows at two flanks (Rouppe van der Voort et al. 2010). Three days later, two umbral cores eventually coalesced into a single umbra (Figs. 1e-g). During this process, the bridge waned and eventually vanished.

It is unusual that a light bridge, which is normally a lowlying feature at photospheric and chromospheric heights, is also observed at coronal temperatures (Matthews et al. 2012). In this case, the light bridge is clearly visible in the $131 \AA, 171 \AA$, $193 \AA$, and $211 \AA$ channels, and marginally seen in the $94 \AA$ and $335 \AA$ bandpasses, so it is very unlikely that the cool components of the response functions made significant contributions to the coronal line emissions (Boerner et al. 2012). Therefore, we believe a significant portion of the plasma of the bridge is heated to coronal temperatures. The coronal component of the bridge is clear of background contamination because the sunspot has an overwhelming positive polarity, and the majority of field lines extend radially, rather than connect to nearby negative polarities to form coronal loops (Fig. 1). Consequently, this could be considered a unique experiment to investigate magnetic field interactions at an intermediate spatial scale.

A one-hour observation was examined starting at 18:30:00 UT 11 July 2012; the data are provided by the Atmospheric Imaging Assembly (AIA; Lemen et al. 2012) on board the Solar Dynamics Observatory (SDO; Pesnell et al. 2012). The analysis concentrates upon the $1600 \AA, 171 \AA$, and $211 \AA$ channels, which have nominal response temperatures of $5000 \mathrm{~K}, 600000 \mathrm{~K}$, and $2000000 \mathrm{~K}$, respectively. The extreme ultraviolet (EUV, $171 \AA$, and $211 \AA$ ) channels both take an image every $12 \mathrm{~s}$; while the UV (1600 $\mathrm{A}$ ) channel captures a frame every 24 s. Level 1 AIA data were obtained and have already been processed by removing the bad pixels and spikes and correcting the flat field. We used the standard routine (prep_aia.pro) in SolarSoft ${ }^{1}$ to calibrate the data into level 1.5. During this step, the data were corrected for the roll angles, interpolated into a common plate scale of $0.6^{\prime \prime}$, and normalized by the exposure time. A small field ofview (FOV; Figs. 1b-d) was traced against solar differential rotation and was co-aligned to a subpixel accuracy.

The magnetograms $\left(B_{z} ;\right.$ Figs. $\left.1 \mathrm{e}-\mathrm{g}\right)$ are provided by the Stokes Spectro-Polarimeter (SP), a component of the Solar Optical Telescope (SOT; Tsuneta et al. 2008) aboard the Hinode satellite (Kosugi et al. 2007). The SOT/SP provides full-Stokes measures of two Fe 1 lines at $630.15 \AA$ and $630.25 \AA$ at the photosphere. Three raster data sets of SOT/SP are used (Fig. 1). The raster scans started at 17:35:28 UT 11 July 2012, 00:47:08 UT 13 July 2012, and 16:00:42 UT 14 July 2012, respectively, and each scan lasted for about $24 \mathrm{~min}$. The raster scans have spatial sampling of about $0.3^{\prime \prime}$ and step size of $0.32^{\prime \prime}$. We used the SOT/SP level 2 data that are the output of full Milne-Eddington inversion $^{2}$ (Lites et al. 2007). The $180^{\circ}$ ambiguity in the azimuthal angle of the magnetic field line is corrected using the AZAM package (Lites et al. 1995).

We use a Cartesian reference frame $[x, y, z]$ with $z$ along the local normal direction. The vertical electric current gives a quantitative measure to the disruption of horizontal magnetic field and is calculated as $j_{z}=\left(\partial B_{y} / \partial x-\partial B_{x} / \partial y\right) / \mu_{0}$ (see the contour in Fig. 3a), where $\mu_{0}$ is the magnetic permeability in free space.

Hinode/SOT Broadband Filter Imager (BFI) provides Ca II $\mathrm{H}$-line observations that are sensitive to emission of plasmas at chromospheric temperatures. Unfortunately, BFI only take images of sunspot AR 11520 at a best cadence of five minutes. Twenty-three images were taken between 16:00-18:10 UT on 11 Jul. 2012, within which six jet-like events were spotted by identifying transient elongated features protruding from the bridge (see method in Louis et al. 2014). Another five events were detected within 27 images captured between 20:20-24:00 UT. The bases of the jet-like activities are indicated in Fig. $3 \mathrm{~d}$.

\footnotetext{
1 http://www.lmsal.com/solarsoft/

2 The level 2 MERLIN inversions are available at https://www2. hao.ucar. edu/csac/csac-data.
} 


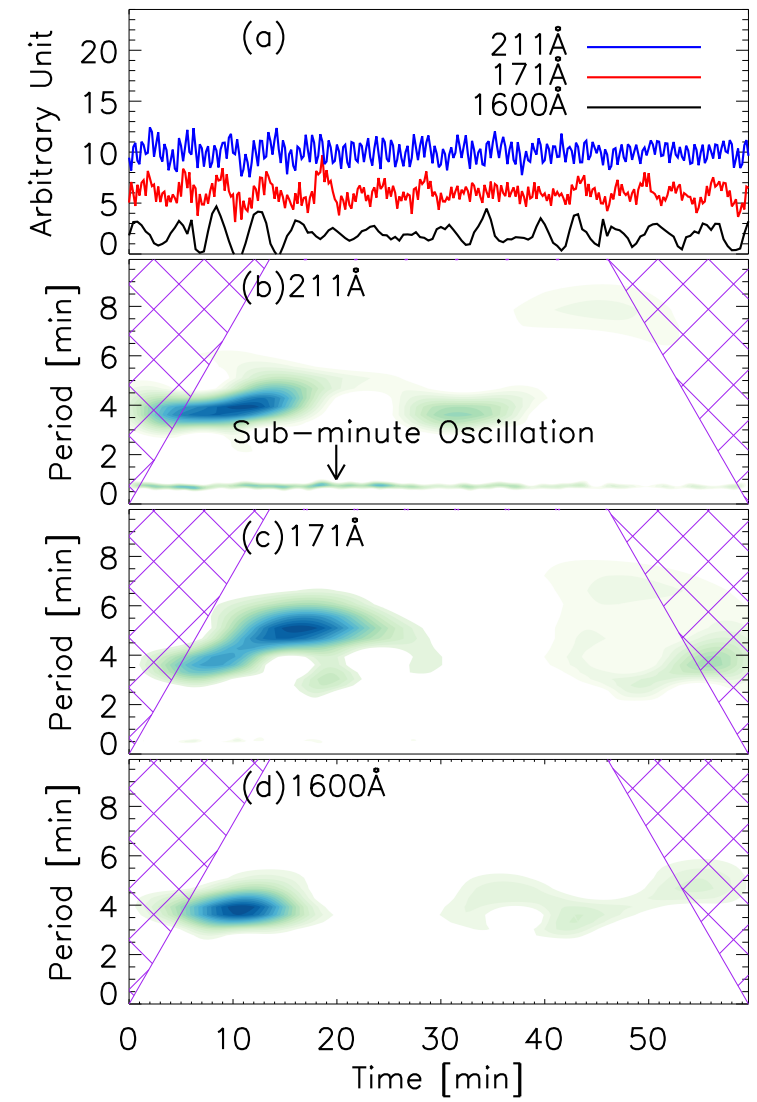

Fig. 2. a) De-trended and normalized emission intensity variations at three AIA bandpasses at the location labelled in Fig. 1. b)-d) Wavelet power spectrum for the $211 \AA, 171 \AA$, and $1600 \AA$ bandpasses, respectively. Only the power spectrum above $90 \%$ confidence level are illustrated. The cross-hatched regions are unreliable owing to zero padding used in the wavelet transform.

\subsection{Time series and spectral analysis}

To illustrate the presence of oscillations and their spectrum dynamics, the emission intensity variations at the western flank of the light bridge were extracted; these are represented by the co-spatial pixels in the $1600 \AA, 171 \AA$, and $211 \AA$ images (the crosses in Figs. 1b-d). The long period trend in each time series was removed by subtracting a moving average of five minute intervals. Then the de-trended time series were normalized by its standard deviation. In Fig. 2a, these time series are plotted subsequently by offsetting their mean values arbitrarily to 2 , 6 , and 10 , respectively, for visualization purposes. The wavelet spectra (Figs. 2b-d) were calculated using the Morlet mother function, which is optimal for illustrating oscillatory processes (Torrence \& Compo 1998).

To investigate the spatial distribution of the oscillatory processes, a power map was constructed for each significant spectral component in a smaller region as enclosed in the rectangle of Fig. 1b. Times series of each pixel was de-trended as in the same manner as for the reference pixel analysed in Fig. 2. The Fourier spectrum was computed using fast Fourier transform. The power was averaged over two bands at $0.5-1 \mathrm{~min}$ and 3-5 min, respectively (see Figs. 3a and d).

To investigate the coherence between different locations, we calculate the cross-correlation coefficient (XCC; Fig. 3b) and lag time (Fig. 3c) between the time series of each pixel and that of the reference pixel (labelled by a cross in Fig. 1b). Only the pixels with oscillation power above the $10 \%$ level relative to the

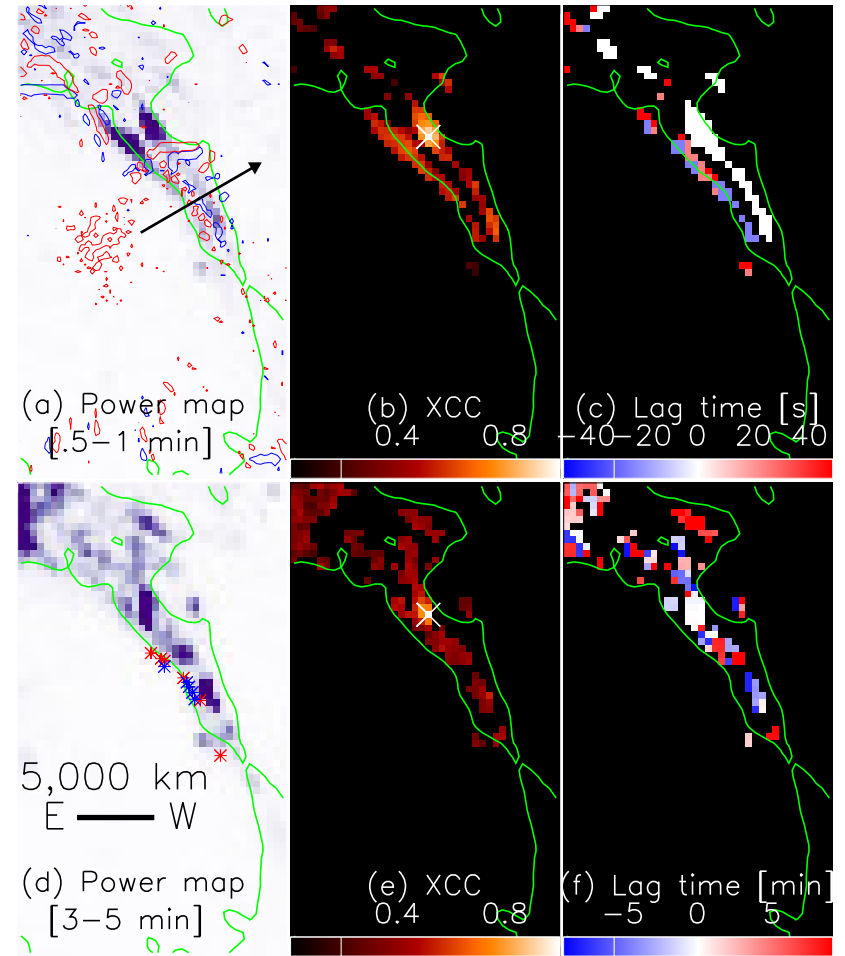

Fig. 3. a), d) Distributions of the Fourier powers of the sub-minute and five-minute oscillations; b), c) distributions of XCC and lag time of the sub-minute oscillations; e), f) are the counterparts of the five-minute oscillations. The green contour in each panel provides a reference of the light bridge structure at the upper photosphere. The red and blue contours in a) labels the electric current density $j_{z}= \pm 40 \mathrm{~mA} \mathrm{~m}^{-2}$, respectively. The red and blue asterisks in d) denotes the base of the jet activities detected at time intervals of 16:00-18:10 UT and 20:20-24:00 UT, respectively.

maximum power of the power map of $0.5-1 \mathrm{~min}$ are considered. The XCCs have a minimum value of about 0.4 , meaning the oscillations are highly correlated within the light bridge.

To study the coherence and lag time between the oscillations at five-minute range, we use the five-point (one minute) running average of the time series, and then recalculate the XCC and lag time (Figs. 3e and f). The running averaging removes the subminute oscillations and, therefore, the XCC and lag time measured in this step reflect the coherence and sequential order of the five-minute oscillations.

\section{Results and discussions}

Light curves of the emission intensity variations are extracted at a representative location of the western flank of the light bridge (Fig. 1). Five-minute oscillations (3-5 min) are significant in all bandpasses considered (Fig. 2); this phenomenon was reported earlier at chromospheric heights by Yuan et al. (2014a) and $\mathrm{Su}$ et al. (2016). Another significant spectral component is detected at $0.5-1 \mathrm{~min}$ (sub-minute oscillations), but only in the $211 \AA$ channel. We also detect significant sub-minute oscillations in the $193 \AA$ channel, and since it is redundant compared to the $211 \AA$ data, we decided not to include it (Fig. 2b). It implies that only the plasmas with temperature between $600000 \mathrm{~K}$ and $2500000 \mathrm{~K}$ are involved. The lack of the sub-minute oscillations in the $1600 \AA$ bandpass could be due to the fact that the $1600 \AA$ channel cannot detect a period shorter than 48 s. However, we did not detect sub-minute oscillations either in the 304 bandpass, 


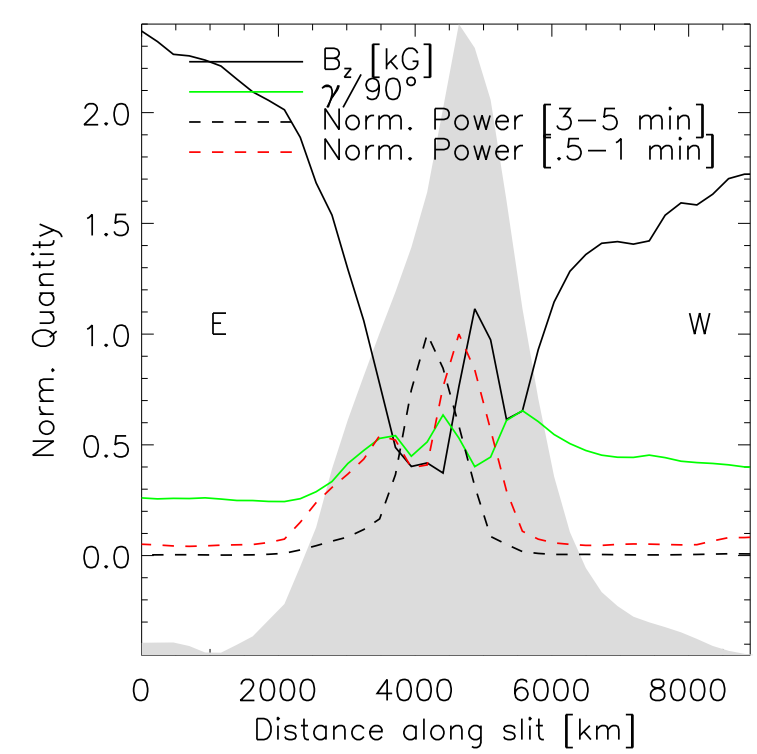

Fig. 4. Profiles of local normal magnetic field strength $B_{z}$, field inclination $\gamma / 90^{\circ}$, and the normalized powers of five and sub-minute oscillations along the arrow labelled in Fig. 3a. The grey shaded region plots the intensity variation of the $1600 \AA$ (scaled to the plot range), and provides a reference for the position of the light bridge.

which has a lower detection limit of $24 \mathrm{~s}$. Therefore, we are convinced that the sub-minute oscillations do not appear in the cool channels.

Figures $3 \mathrm{a}$ and $\mathrm{d}$ shows the spatial distributions of the subminute and five-minute oscillations measured in the $211 \AA$ channel. The five-minute oscillations are distributed along the spine of the light bridge, whereas the sub-minute oscillations are distinctively co-spatial with the two flanks of the bridge. This strongly indicates that the driver for these sub-minute oscillations is likely to be connected with the collective interactions between magnetic field of the bridge and the sunspot.

Figure $3 \mathrm{~b}$ shows that the sub-minute oscillations are highly correlated with each other; the XCC is generally greater than 0.4 . Figure $3 \mathrm{c}$ illustrates that the sub-minute oscillations along the western flank of the bridge have zero time lag. Therefore, it could be postulated that these emission intensity variations are driven by surface-to-surface buffering, rather than random interactions of adjacent fieldlines along that flank. The emission intensity variations at the eastern flank respond either $24 \mathrm{~s}$ ahead or after the signal of the western flank. Two pairs of surface-tosurface interactions may exist at the eastern flank, however, the AIA resolution does not allow a conclusive distinction.

Therefore, it can be inferred that the sub-minute oscillation at two ridges could have the same driver with regard to the periodicity, as they are highly correlated. However, the processes occurring at two flanks might be different based on the fact that the lag time distribution shows asymmetry in the spatial domain. It should be noted that there is some weak evidence that the eastern flank is surrounded by isolated islands of strong electric current, while the western flank is relatively clearer of such islands (Fig. 3a). Moreover, the jet-like activities are only detected at the eastern flank ${ }^{3}$ (Fig. 3d), which means magnetic reconnection is repetitively triggered there.

\footnotetext{
3 Although SOT/BFI observations did not cover the AIA observation interval used in this study, we don't think the spatial distribution of the jet activities will change within a timescale of hours.
}

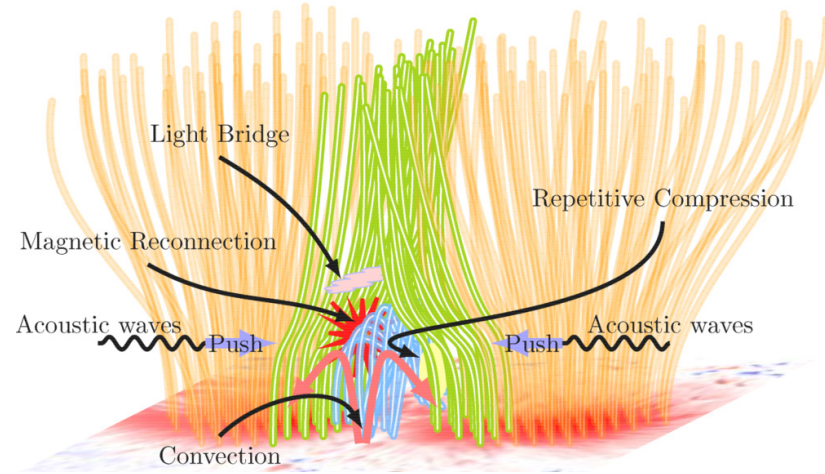

Fig. 5. Sketch illustrating the magnetic morphology and dynamics. The magnetic field (sky blue) of the light bridge is weakly twisted and intrudes into the sunspot. The field lines (dark green) sandwiching the light bridge are dragged by the downward motions of partial convections at two flanks of the bridge and become more inclined. Compression and rarefaction dominate the western flank, while magnetic reconnection is triggered at the eastern flank.

Figure 4 plots the profiles of local normal magnetic field strength $B_{z}$, the field inclination $\gamma$, and the normalized powers of the sub- and five-minute oscillations across the bridge, as labelled by an arrow in Fig. 3a. Both profiles of $B_{z}$ and $\gamma$ have two local valleys across the bridge. At the western flank, the decrease (increase) of $B_{z}$ is associated with the increase (decrease) of $\gamma$. This anti-correlation is expected if we consider that $B_{z}=B \cos (\gamma)$ for $\gamma \in\left[0,90^{\circ}\right]$. In contrast, at the eastern flank, such an anti-correlation does not hold, meaning that a more complex magnetic structure may have formed and that the field strength and azimuthal angle also have significant influence on the structure. This finding supports the asymmetries found in the spatial pattern of sub-minute oscillations and jet activities. However, at the current stage, we cannot reach a better conclusion with limited information.

Figures $3 \mathrm{e}$ and $\mathrm{f}$ shows the $\mathrm{XCC}$ and lag time of the fiveminute oscillations. In contrast, the XCC is relative weaker, and the lag time forms localized patches, which means the coherence length is much shorter than that of sub-minute oscillations. Yuan et al. (2014a) found that five-minute oscillations are almost in phase at chromosphere, so it is still unknown why the five-minute oscillations lose spatial coherence when observed in coronal lines.

Recent simulations and observations of Toriumi et al. (2015a,b) show that the magnetic field of a light bridge is twisted and related to an emerging dipole field (also supported by Louis et al. 2015). Based on this knowledge and our observations, we propose a scenario to explain these results as depicted in Fig. 5. The sunspot has a predominant radially expanding magnetic field; the magnetic field of the light bridge is slightly twisted and intrudes into the umbral field. The buffering between two magnetic fields are driven by any dynamics of the ambient fluid: convections, granulation, turbulence, seismic waves, jet activities, self-organized dynamics, etc. At the western flank, the magnetic field of the bridge does not reverse direction relative to the umbral field and, therefore, compression and rarefaction between two fields dominate and cause temperature perturbations. In contrast, at the eastern flank the field is likely to be relatively more reversed compared with the magnetic field of the umbra and form an X-point where magnetic reconnection could occur (Cheung \& Cameron 2012). This kind of asymmetry between two flanks of a light bridge is also consistent with fact that the chromospheric jet activities only cluster at one flank 
of the light bridge (see Fig. 3d). Small-scale flux emergence and jet activities are also reported in other granular light bridge examples; these studies support the concept of an asymmetry of two flanks of a bridge (Louis et al. 2014, 2015).

In the speculated scenario, magnetic reconnections are triggered recurrently at the eastern flank, while repetitive compressions of magnetic field are driven by the same source at the western flank. This kind of magnetic morphology has been examined numerically with repetitive reconnections triggered subsequently (Shibata et al. 1992; Archontis \& Hood 2013). These recurring small-scale magnetic reconnections might be responsible for the waning of the bridge: magnetic ablation by reconnection could eventually lead to the disappearance of the bridge in a few days. Figure $2 \mathrm{~b}$ shows that the sub-minute oscillation power become weaker during this observational period. We will investigate this possible phenomenon in follow-up studies.

With regard to the periodicity at $0.5-1 \mathrm{~min}$, source evidence is inconclusive in our current study. One possible source is the eigen mode of the magnetic resonator. Magnetic null point could host oscillatory reconnections due to plasma overshots. Oscillation timescale is estimated to be $\tau_{\text {osc }}=2 \ln S$ in unit of Alfvén transit time from outer boundary into the diffusion region (Craig \& McClymont 1991), where Lundquist number $S$ is of the order of $10^{6}$ at chomospheric height. The Alfvén transit time is estimated as $0.1 L / V_{\mathrm{A}}$, where $L=100 \mathrm{~km}$ is the typical length scale of the reconnection site, where $0.1 L$ is the length estimate of diffusion region (Craig \& McClymont 1991), and $V_{\mathrm{A}}=10 \mathrm{~km} \mathrm{~s}^{-1}$ is a typical Alfvén speed at chromospheric height. The magnetic resonator filters out the resonance period from the random perturbations (McLaughlin et al. 2009, 2012). In our case, we estimate the oscillatory timescale at $\tau_{\text {osc }} \sim 30 \mathrm{~s}$ (see Craig \& McClymont 1991, for method), which is consistent with our measurement.

In the proposed scenario, a dominant polarity is evaded by an emerging magnetic dipole. Such magnetic configurations have analogous models, such as the triggering mechanisms of X-ray jets (Archontis \& Hood 2013; Sterling et al. 2015), flares (Shibata \& Magara 2011), and mass ejections (Chen 2011), at both smaller and larger scales. In our case, the reconnection is proposed to be triggered in a gentle and continuous manner; it also provides insight into the structure of the light bridge and the waning process (continuous ablation by repetitive magnetic reconnections).

This scenario also supports the nanoflare or microflare model. In the case of coronal loops, nanoflare heating (Parker 1988 ) is proposed to occur rapidly at a spatial scale of $10 \mathrm{~km}$ or smaller, but it cannot be observed directly with current instruments. In the case of a sunspot contaminated by a light bridge, the interaction scale is up to the scale of $1000 \mathrm{~km}$ and is within the resolution capability of available instruments. In this observation, a light bridge, which is normally a low atmosphere (low temperatures of $100000 \mathrm{~K}$ or less) feature, is unusually observed at coronal channels (at about $2000000 \mathrm{~K}$ ) for hours. Continuous heating is required to maintain the plasma at coronal temperatures. Moreover, we detected short-period (30-60 s) oscillations in the emission intensity only at hot coronal channels, with the timescale consistent with the high-frequency heating model (Klimchuk 2015).

This study reports the first detection of two abnormal oscillation modes in a waning light bridge; our findings suggest a variety of new features. However, several of these new features cannot be conclusive owing to limited spatial and temporal resolutions. Coordinated observations with currently available instruments should be sufficient to provide better insights, for example New Solar Telescope (NST), Interface Region Imaging Spectrograph (IRIS). We note that although dynamic activities are prevailingly detected at two flanks of a light bridge, the spine usually looks brighter in UV and EUV bandpasses. This might be related to the complex magnetic structure of the light bridge.

\section{References}

Archontis, V., \& Hood, A. W. 2013, ApJ, 769, L21

Boerner, P., Edwards, C., Lemen, J., et al. 2012, Sol. Phys., 275, 41

Borrero, J. M., \& Ichimoto, K. 2011, Liv. Rev. Sol. Phy., 8, 4

Botha, G. J. J., Arber, T. D., Nakariakov, V. M., \& Zhugzhda, Y. D. 2011, ApJ, 728,84

Chae, J., \& Goode, P. R. 2015, ApJ, 808, 118

Chen, P. F. 2011, Liv. Rev. Sol. Phys., 8, 1

Cheung, M. C. M., \& Cameron, R. H. 2012, ApJ, 750, 6

Craig, I. J. D., \& McClymont, A. N. 1991, ApJ, 371, L41

Jess, D. B., De Moortel, I., Mathioudakis, M., et al. 2012, ApJ, 757, 160

Jess, D. B., Reznikova, V. E., Van Doorsselaere, T., Keys, P. H., \& Mackay, D. H. 2013, ApJ, 779, 168

Jess, D. B., Reznikova, V. E., Ryans, R. S. I., et al. 2016, Nature Phys., 12, 179 Khomenko, E., \& Collados, M. 2015, Liv. Rev. Sol. Phys., 12, 6

Klimchuk, J. A. 2015, Philosoph. Trans. Roy. Soc. Lond. Ser. A, 373, 20140256 Kosugi, T., Matsuzaki, K., Sakao, T., et al. 2007, Sol. Phys., 243, 3

Kwak, H., Chae, J., Song, D., et al. 2016, ApJ, 821, L30

Lagg, A., Solanki, S. K., van Noort, M., \& Danilovic, S. 2014, A\&A, 568, A60 Lemen, J. R., Title, A. M., Akin, D. J., et al. 2012, Sol. Phys., 275, 17

Lites, B. W., Low, B. C., Martinez Pillet, V., et al. 1995, ApJ, 446, 877

Lites, B., Casini, R., Garcia, J., \& Socas-Navarro, H. 2007, Mem. Soc. Astron. It., 78, 148

Louis, R. E., Bellot Rubio, L. R., Mathew, S. K., \& Venkatakrishnan, P. 2009, ApJ, 704, L29

Louis, R. E., Beck, C., \& Ichimoto, K. 2014, A\&A, 567, A96

Louis, R. E., Bellot Rubio, L. R., de la Cruz Rodríguez, J., Socas-Navarro, H., \& Ortiz, A. 2015, A\&A, 584, A1

Matthews, S., Baker, D., \& Domínguez, S. V. 2012, in Hinode-3: The 3rd Hinode Science Meeting, eds. T. Sekii, T. Watanabe, \& T. Sakurai, ASP Conf. Ser., 454, 209

McLaughlin, J. A., De Moortel, I., Hood, A. W., \& Brady, C. S. 2009, A\&A, 493, 227

McLaughlin, J. A., Thurgood, J. O., \& MacTaggart, D. 2012, A\&A, 548, A98

Muller, R. 1979, Sol. Phys., 61, 297

Parker, E. N. 1988, ApJ, 330, 474

Pesnell, W. D., Thompson, B. J., \& Chamberlin, P. C. 2012, Sol. Phys., 275, 3

Reznikova, V. E., \& Shibasaki, K. 2012, ApJ, 756, 35

Rimmele, T. 2008, ApJ, 672, 684

Rimmele, T. R. 1997, ApJ, 490, 458

Rouppe van der Voort, L., Bellot Rubio, L. R., \& Ortiz, A. 2010, ApJ, 718, L78

Schüssler, M., \& Vögler, A. 2006, ApJ, 641, L73

Shibata, K., \& Magara, T. 2011, Liv. Rev. Sol. Phys., 8, 6

Shibata, K., Nozawa, S., \& Matsumoto, R. 1992, PASJ, 44, 265

Snow, B., Botha, G. J. J., \& Régnier, S. 2015, A\&A, 580, A107

Sobotka, M., Bonet, J. A., \& Vazquez, M. 1994, ApJ, 426, 404

Sterling, A. C., Moore, R. L., Falconer, D. A., \& Adams, M. 2015, Nature, 523, 437

Su, J. T., Ji, K. F., Banerjee, D., et al. 2016, ApJ, 816, 30

Toriumi, S., Cheung, M. C. M., \& Katsukawa, Y. 2015a, ApJ, 811, 138

Toriumi, S., Katsukawa, Y., \& Cheung, M. C. M. 2015b, ApJ, 811, 137

Torrence, C., \& Compo, G. P. 1998, Bull. Am. Meteorological Soc., 79, 61

Tsuneta, S., Ichimoto, K., Katsukawa, Y., et al. 2008, Sol. Phys., 249, 167

Yang, S., Zhang, J., Jiang, F., \& Xiang, Y. 2015, ApJ, 804, L27

Yuan, D., \& Van Doorsselaere, T. 2016a, ApJS, 223, 23

Yuan, D., \& Van Doorsselaere, T. 2016b, ApJS, 223, 24

Yuan, D., Nakariakov, V. M., Huang, Z., et al. 2014a, ApJ, 792, 41

Yuan, D., Sych, R., Reznikova, V. E., \& Nakariakov, V. M. 2014b, A\&A, 561, A19

Yuan, D., Su, J., Jiao, F., \& Walsh, R. W. 2016, ApJS, 224, 30

Zhugzhda, Y. D. 2008, Sol. Phys., 251, 501 